\title{
Isolation in Coordination: Challenges of Caregivers in the USA
}

\author{
Mark W. Schurgin \\ Google Health \\ Palo Alto, USA \\ schurgin@google.com
}

\author{
Mark S. Schlager \\ Google Health \\ Palo Alto, USA \\ mschlager@google.com
}

\author{
Laura M. Vardoulakis \\ Google Health \\ Palo Alto, USA \\ lauravar@google.com
}

\author{
Laura R. Pina \\ Google \\ Mountain View, USA \\ laurapina@google.com
}

\author{
Lauren G. Wilcox \\ Google \\ Mountain View, USA \\ lwilcox@google.com
}

\begin{abstract}
As the global population ages and the prevalence of chronic conditions and acute infections rise, it is becoming imperative to understand the many forms of caregiving labor and create sociotechnical systems that support them. In this paper, we report results of a large survey study with 2000 informal caregivers in the USA, highlighting the fundamental challenges that different types of caregivers face when coordinating care with others. Our findings support previous findings on caregivers' coordination challenges, while also offering insights into the situational, mediating factors that influence the extent to which care coordination challenges are felt. These mediating factors include caregivers' relationships, access to a variety of resources including physical, social, and financial support, and physical and mental barriers. We discuss these challenges and mediating factors, and conclude with a discussion on how they can be considered in the design of future sociotechnical systems.
\end{abstract}

\section{CCS CONCEPTS}

- Human-centered computing $\rightarrow$ Empirical studies in collaborative and social computing;

\section{KEYWORDS}

Caregiving, Health, Well-being, Care Coordination, Care Communication

\section{ACM Reference Format:}

Mark W. Schurgin, Mark S. Schlager, Laura M. Vardoulakis, Laura R. Pina, and Lauren G. Wilcox. 2021. Isolation in Coordination: Challenges of Caregivers in the USA. In CHI Conference on Human Factors in Computing Systems (CHI '21), May 8-13, 2021, Yokohama, fapan. ACM, New York, NY, USA, 14 pages. https://doi.org/10.1145/3411764.3445413

\footnotetext{
Permission to make digital or hard copies of all or part of this work for personal or classroom use is granted without fee provided that copies are not made or distributed for profit or commercial advantage and that copies bear this notice and the full citation on the first page. Copyrights for components of this work owned by others than ACM must be honored. Abstracting with credit is permitted. To copy otherwise, or republish, to post on servers or to redistribute to lists, requires prior specific permission and/or a fee. Request permissions from permissions@acm.org.

CHI '21, May 8-13, 2021, Yokohama, Japan

(C) 2021 Association for Computing Machinery.

ACM ISBN 978-1-4503-8096-6/21/05 ..\$15.00

https://doi.org/10.1145/3411764.3445413
}

\section{INTRODUCTION}

Informal caregivers provide ongoing, unpaid care for another individual - typically family members or friends - with a chronic condition or disability $[44,46,65]$. Their responsibilities vary greatly, from checking in on care recipients, to managing engagements with the healthcare system, to assisting with activities of daily living, such as bathing, eating, and online activities [24, 39, 53]. Informal caregivers play a vital role in the larger care team, directly performing or influencing work that shapes the recipients' day-to-day life [10].

The need to understand and support caregiving work is becoming urgently important: the percentage of adults age 65 and older has risen in countries across the globe, with large increases in Japan, Italy, Portugal, France, Germany, Denmark, UK, USA, among many others [4]. In parallel, the prevalence of chronic conditions and acute infections are on the rise [48]. In the USA, the aging baby boomer generation, rising prevalence of chronic conditions, and longer lifespans indicate an increased need to support the caregiving of older adults [10]. Furthermore, a variety of factors, such as a shortage of medical professionals and decreased hospital stays, have shifted the burden of care from the traditional healthcare system to informal caregivers [18].

These changes are driving societal awareness of informal home care, with estimates that more than 1 in 5 Americans $(21.3 \%$ or 53 million) are caregivers of adults or children with special needs. These estimates are up from the estimated 43.5 million U.S. caregivers in 2015 [10]. Unfortunately, informal caregivers' health can suffer as care work is often mentally and physically demandingconsistently so-and work typically goes unrecognized [23, 65].

The number and types of relationships between caregivers and their care recipient(s) are wide-ranging, leading to a large body of past research in $\mathrm{HCI}$ focused on specific caregiving relationships. This research spans parental caregivers of children with chronic conditions [2, 28, 29, 31, 32], caregivers of family members with depression $[73,75]$, friends and family supporting veterans with PTSD [19], volunteers' caregiver knowledge and its role in dementia care [21], and spousal caregivers [5, 66], among many others.

Across these diverse relationships, caregivers must coordinate care, often with both the care recipient and other formal and informal caregivers. Care coordination challenges can vary relative to the specific dynamics of these relationships and caregiver type. For example, in the case of spousal caregiving, when the values between a spousal caregiver and their recipient are asymmetric, perceived 
tensions increase and the ability to coordinate care decreases [5]. Caregivers of family members often assume implicit roles to fulfill their duties, resulting in invisible labor when coordinating with others, which can increase conflict with other family members [25]. Finally, parents caring for high-risk infants face multiple barriers to communication and care coordination, such as the massive amounts of data and information provided by medical professionals, which interferes with caregivers obtaining a holistic view of the health of their child [35].

While it is vital to understand how specific caregiving relationships and situations give rise to specific challenges, research on common challenges and needs spanning informal caregiver groups is just beginning [65]. Establishing this understanding will be useful for understanding which aspects of caregiving are broadly applicable across different groups, but also for identifying when specific group dynamics for different kinds of caregivers need to be taken into account.

The research we present here seeks to address this gap, through a large survey of 2000 informal caregivers in the USA. The overarching questions motivating our survey study include:

(1) Where do commonalities exist across the types of work that informal caregivers perform?

(2) What are these informal caregivers' primary care coordination challenges, and how do they compare to the challenges we've learned about through prior, deeper, qualitative studies?

(3) Which coordination challenges could be addressed through the evolution of sociotechnical systems, and how might we think about design to address these challenges?

In this paper, we make the following contributions to $\mathrm{HCI}$ :

(1) A snapshot of informal caregivers in the USA, including descriptive findings on the kinds of care work and tasks they perform, based on a large-scale survey of 2000 informal caregivers.

(2) Description of the fundamental challenges that varied types of caregivers face when coordinating care with others, which supports the findings of prior, qualitative studies on informal care work.

(3) Description of the mediating factors that impact the fundamental challenges of care coordination, based on a comprehensive, qualitative analysis of open-ended survey responses.

\section{RELATED WORK}

\subsection{Studies of Informal Caregivers}

Caregiving has been the focus of many recent studies in HCI. These studies have contributed to a host of areas in our field: design and prototype deployment research for technologies that support aspects of informal caregiving [14, 40, 42, 74], analyses of the use of existing technologies to support caregiving [66], patient self-care in collaboration with caregivers [43], and foundational qualitative studies of people's experiences with caregiving [12, 24, 25, 39]. We focus our discussion of related work on the latter-foundational qualitative studies-as we seek to build on and extend understandings of caregivers' primary challenges in coordinating care.

\subsection{Informal Caregivers' Work}

Chen et al.'s foundational study set the stage for a stream of research identifying common challenges and coping strategies of caregivers [12]. Noting that many current health and well-being applications are clinician- or patient-centric in design, Chen et al. shifted the analytic lens to other caregivers' needs and work, which includes managing, coordinating, and handling physical activities. These activities have emotional and social consequences, which can be negative, as well as reflective consequences, which can be positive. Chen and colleagues made specific design recommendations including supporting coordination among care and personal tasks, managing social-emotion work, and encouraging reflection and learning [12].

Indeed, caregivers conduct many different types of work in their caregiving activities, and further inquiry around types of work has led to numerous insights. For example, researchers have introduced infrastructuring work to describe patients' and caregivers' dynamic and ongoing work to assemble and (re)-configure multiple, often fragmented services, in order to access them in concert [13, 24]. This assembly and reconfiguration involves connecting and managing human and non-human entities (e.g., physical and digital materials and artifacts, people, places) both within and beyond healthcare settings. Part of infrastructuring work involves aligning components of care infrastructures, to mend breakdowns that pose barriers and gaps to care processes and resources. Alignment work can concern both the temporal and material domains.

\subsection{Informal Home Care Work}

For home care work specifically, Bratteteig and Eide identify seven types of work: information work, articulation work, illness work, everyday life work and life-changing work as well as activities concerned with the interpersonal work of relationship building: relation work and discretion work [6]. To distill these types, they drew on a large body of research (e.g., [1, 3, 34, 41, 50]) and grounded their auto-ethnographic fieldwork in care work frameworks [15, 63].

Currin et al.'s interview study with primary family and respite caregivers also identified the importance of interpersonal work [16]. They found that "building trust" was a core need, and one that could be strengthened with status updates, learning routines and care management, and technology access (cohering broadly with Bratteteig and Eide's informaton, articulation, and everyday life work). This theme of building and maintaining trust and supporting relation work was also a prominent finding in Singh et al.'s study on informal caregivers in the New York City area [59].

Looking across the research to distill types of care work, Chen et al.'s [12] work themes (managing, coordinating, handling physical activities) cut across the more nuanced work types introduced above. For example, life-changing work [6]-which draws from Corbin and Strauss' concept of biographical work [15] to manage identity adaptation-requires that caregivers provide physical activity support with tasks (e.g., dressing) while managing knowledge and information about which tasks need assistance, and finally, coordinating with other caregivers and the recipient to balance caregiver support with recipient autonomy.

In parallel to research focused on defining care work, complementary research has examined the needs of home care workers 
more broadly. Through an ethnographic study including probes in ten homes, Schorch and colleagues [57] studied informal, domestic caregivers who are also elderly. They identified key challenges of structuring care agendas and managing time in their coordination activities, as well as vital needs for social support, including sharing experiences and information, and identifying common problems and solutions. Tixier's study on the social support activities of older informal caregivers found that support for reconciling online and offline experiences is an important area for further study [67].

\subsection{Informal Care Coordination Work}

Informal caregivers often manage communication and coordination with a complex social group, including friends, family, and other formal and informal caregivers. This includes the need to maintain awareness of and also negotiate the level of involvement of each individual involved in the recipients' care, which can be dynamic $[14,65]$.

Looking across several informal caregiver types, Tang et al.'s qualitative study with 15 informal caregivers identified the main challenges they faced, mainly: maintaining awareness, coordinating hand-offs among informal caregivers, and easing tensions among caregivers and patients. They found that primary caregivers not only coordinated all the necessary, interleaved care tasks, they also led articulation work [56, 58].

Gutierrez and colleagues' cross-generational study of informal elderly caregiving in Chile and Argentina found that family members assumed implicit roles for caregiving, without formally communicating, coordinating, or making visible the commitments they associated with their role. These implicit role assumptions strained caregiver relationships by increasing conflicts and introducing inefficiencies [25]. These findings suggest a need to support articulation work in elderly caregiving. Indeed, we see the recurring need to support articulation work in Tang et al.'s findings, Bratteteig's identified types of care work [6], and in Gutierrez and colleagues' findings [25], among others. Taken together, findings of these studies also echo the large role of interpersonal work and its intersections with articulation and information work.

Looking specifically at the role of technology in coordination, research has investigated how to better support situational, collaborative awareness of care activities. Yamashita et al. explored the role of artifacts in care coordination, studying caregivers' sharing of journals and information about their recipients, anonymously with other caregivers. While they found positive effects for caregivers and recipients, a consequence of information sharing included competition among caregivers and suppression about joyous events [75]. In the context of family with young children or teenagers, recent work has demonstrated that: first, sociotechnical systems to support the health management of care recipients can distribute activities between caregivers and care recipients, and second, care recipients (children), preferred to use technology to mediate communication with their caregivers (parents) when it came to sharing information about their symptoms and health management $[27,51,52]$.

While the prior research provides important foundations on which to build, investigations into the specific challenges of informal caregivers is a rather new area of inquiry in HCI [65]. We provide the first survey study-of which we are aware-that examines how informal care work and coordination challenges are experienced across a large, broad swath of informal caregivers in the USA, representing many different caregiving relationships, recipient needs, and life situations.

\section{SURVEY STUDY}

\subsection{Survey Design}

Our survey study collected data through a commercial web-based survey service, with targeted participant recruitment (i.e., survey panel), following a purposeful sampling approach, using criterion sampling [47]. Participants were recruited using the survey company's established methods [69], applying inclusion criteria described below. At the beginning of the survey, participants were reminded that the survey would ask health-related questions and asked if they would like to continue. Participants who said no were excluded. The survey instrument did not collect individually identifiable information.

The survey featured a total of 57 questions, including demographic and relationship information of the respondent, care recipient, and other caregivers, caregiver tasks and duties, time spent providing care, technologies used in caregiving and coordinating care, the effects of caregiving on health and well-being, and challenges coordinating care. ${ }^{1}$ In this paper, we primarily report on results from the survey questions that centered on care coordination, including:

- In the past 30 days, how have you coordinated caregiving with another person?

- In the past 30 days, how frequently have you coordinated caregiving with another person?

- In the past 30 days, which tools have you used to coordinate schedules, tasks, and hand-offs with other caregivers?

- What are your biggest challenges or difficulties in coordinating care with another person? [opened-ended]

Before deployment, we confirmed the face validity of the online instrument to ensure that the assessment items were appropriate to the targeted constructs and study objectives. Survey testers included researchers with expertise in literacy and survey design. All testers took the survey multiple times with different scenarios in mind, to ensure completeness of response sets and provided feedback on clarity of wording, layout and order of questions, and perceived ability to answer them. Findings from these tests allowed us to improve the survey design in an iterative fashion before deployment. The survey service launched the survey with $10 \%$ of the total sample size first, pausing to verify data integrity and expected completion time, before full deployment. No changes were needed based on this "soft launch". Thus, this soft launch data were incorporated into the final sample.

\subsection{Recruitment}

We surveyed a purposeful sample of 2000 informal caregivers across the USA. Though our sample was not fully representative of all caregiver populations (e.g., we did not include non-English-reading

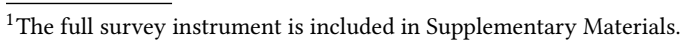


caregivers), we set our inclusion criteria to strive for varied representation of caregiving relationships, as follows:

- Must be able to read English

- Must be willing to answer health-related questions

- Must be 18 years old or older

- Must use a smartphone as their personal mobile device

- Must identify as helping another adult individual with Activities of Daily Living (ADLs), Instrumental Activities of Daily Living (IADLs), or some general social/emotional activity (e.g., regular visits or phone calls)

- Cannot identify as a professional (paid) caregiver

- Care recipient must live at home or in an independent community (no professional onsite staff)

As we were not interested in assessing the prevalence of caregivers in the USA, we instead sought a sample that is representative of core groups of caregivers previously identified in HCI research, and elicited information about these caregivers' behaviors and characteristics.

To make sure our sample remained representative of different types of caregivers, during data collection we enforced quotas for target age groups and to set limits on the number of men and women, and the number of high-income earners. Table 1 reports details of our quotas, which included:

- Gender: No more than 50\% men, no more than $70 \%$ women.

- Age: Quotas match representative caregiving age groups [10].

- Overall sample representative geographically of the U.S. population.

- Overall sample representative socioeconomically (by region) of the U.S. population.

Upon completion, respondents were compensated approximately $\$ 10$ USD. The completion time for the survey was approximately 15 to 20 minutes.

\subsection{Survey Data Analysis}

Throughout survey data collection, the survey provider monitored response data for completeness, quality, and duplicates. In total, 580 responses did not meet our inclusion criteria (Section 3.2), and were subsequently replaced, yielding a total of 2000 final responses.

We analyzed survey questions with single-selection and multipleselection responses using descriptive statistics. Our analysis of closeended questions (examples given in Section 3.1) was theoretically driven (top-down), in accordance with question design, considering Corbin and Strauss' Illness Trajectory Theory and lines of work: illness work, everyday life work, and biographical work [15].

The open-ended question we report on (listed in Section 3.1 and reported on in Section 4.5) was analyzed inductively. For our inductive analysis, four authors independently reviewed each openended response comprising the final data set. All four engaged in deep and prolonged data immersion, discussion, comparison of interpretations of the meaning of the data, and reflection. We met in multiple rounds to collaboratively revise and refine codes for response data. All disagreements were resolved through collaborative rounds of review among researchers, in synchronous discussion.
Finally, the original four researchers met in multiple rounds to generate domain categories and themes, through deep reflection on data and relationships among codes, reflexively $[7,8]$.

\section{FINDINGS}

We first describe our sample of informal caregivers and follow this with a summary of their caregiving relationships. We provide an overview of their caregiving activities, and then dive more deeply into their specific care coordination work, focusing on the primary challenges they face when coordinating care with another person.

\subsection{Survey Sample}

Here we report on survey respondent demographics to illustrate the varied life situations of our participants. Table 2 reports comprehensive participant statistics on age, gender, ${ }^{2}$ and income level. The final sample of 2000 respondents compared well with the U.S. population in geographic diversity and socioeconomic variability. Consistent with complementary research on caregiver demographics and our quotas, the sample skewed toward women (70.9\%). With respect to age, under $5 \%$ of respondents were between the ages of 18-24, while over half of the respondents were 55 or over [10].

With respect to type of health insurance the care recipient received, we found four categories: Medicaid, Medicare, private insurance, and Veterans health care (see Table 4). Medicaid is a healthcare program funded jointly by states and the federal government of the U.S. Medicaid provides healthcare coverage to low-income adults, children, pregnant women, elderly adults and people with disabilities. As of mid-year in 2020, Medicaid covered 66.8 million Americans [37]. Medicare is a federal health insurance program for people who are 65 years old or older, people with certain disabilities, and those with end-stage renal disease [38]. Approximately, 44 million Americans receive Medicare [30]. Veterans health care is benefit program for American military veterans, that provides access to a national, integrated health care system (the Veterans Health Administration). This healthcare system is the largest integrated healthcare system in the U.S. and provides care for approximately nine million veterans [45]. Respondents could select more than one type of insurance.

For care recipients under the age of $65,39 \%$ receive Medicaid, $24 \%$ receive Medicare, and $18 \%$ have private insurance. For care recipients over 65 years of age, $13 \%$ receive Medicaid, $64 \%$ receive Medicare, and $14 \%$ have private insurance. For both age ranges, $5 \%$ or less receive Veterans health care insurance.

We asked respondents to describe where they live with respect to type of region: small town or rural, suburban, or urban. Thirty-two percent of respondents self-identified as living in small town or rural, while $48.9 \%$ said they live in suburban region, and $19.2 \%$ said they live in an urban region. With respect to region in the country, $19.9 \%$ were from the Midwest, $20.1 \%$ were from the Northeast, $40 \%$ were from the South, and $20.2 \%$ were from the West of the U.S. (based on U.S. Census regions) [9].

\footnotetext{
${ }^{2}$ We report on all selected gender identity data in Table 2 . This survey was designed and deployed in 2019 and the label "Other" is used to elicit non-binary status or the preference to self-describe, no longer best practice. We now recommend the approaches published in 2019 by Spiel et al. [61] to collect gender identity data.
} 
Table 1: Survey gender quotas (left), age range quotas (center), and income upper limit (right)

\begin{tabular}{cc|crr|rr}
\hline Gender & Limit $n(\%)$ & Age & Target $\approx n$ & $(\%)$ & Income Bracket & Limit $n(\%)$ \\
\hline Women & $\leq 1400(70)$ & $18-24$ & 100 & $(5)$ & $\geq 100 \mathrm{k}$ & $\leq 600(30)$ \\
Men & $\leq 1000(50)$ & $25-44$ & 500 & $(25)$ & & \\
& & $45-64$ & 900 & $(45)$ & & \\
& & $65+$ & 500 & $(25)$ & & \\
\hline
\end{tabular}

Table 2: Respondents' self-reported gender (left), age (center), and highest level of education completed (right) $(n=2000)$.

\begin{tabular}{crc|rrr|crr}
\hline Gender & $n$ & $(\%)$ & Age & $n$ & $(\%)$ & Level of Education & $n$ & $(\%)$ \\
\hline Women & 1418 & $(70.9)$ & $18-24$ & 92 & $(4.60)$ & No High School diploma & 39 & $(1.95)$ \\
Men & 578 & $(29.0)$ & $25-34$ & 209 & $(10.45)$ & High school diploma & 349 & $(17.45)$ \\
Non-binary/ & & & $35-44$ & 281 & $(14.05)$ & Some college or & & \\
Other & 2 & $(<1.0)$ & $45-54$ & 247 & $(12.35)$ & certification & 787 & $(39.50)$ \\
Prefer not & & & $55-64$ & 667 & $(33.35)$ & Bachelor's Degree & 490 & $(24.50)$ \\
to say & 2 & $(<1.0)$ & $65-74$ & 431 & $(21.55)$ & Professional Degree, & & \\
& & & $75-84$ & 69 & $(3.45)$ & Masters, or Doctorate & 335 & $(16.74)$ \\
& & & $85+$ & 4 & $(0.20)$ & & & \\
\hline
\end{tabular}

Table 3: Respondents' current state of employment (left) and annual household income (right) $(n=2000)$.

\begin{tabular}{crr|crrr}
\hline State of employment & $n$ & $(\%)$ & Annual household income (USD) & $n$ & $(\%)$ \\
\hline Retired & 632 & $(31.60)$ & $<10,000$ & 83 & $(4.15)$ \\
Employed full-time & 523 & $(26.60)$ & $10,000-19,999$ & 131 & $(6.55)$ \\
Employed part-time & 268 & $(13.40)$ & $20,000-34,999$ & 334 & $(16.70)$ \\
Homemaker & 179 & $(8.95)$ & $35,000-49,999$ & 318 & $(15.90)$ \\
Self-employed & 129 & $(6.45)$ & $50,000-74,999$ & 444 & $(22.20)$ \\
Unable to work & 112 & $(5.60)$ & $75,000-99,999$ & 313 & $(15.65)$ \\
Unemployed/ looking & & & $99,999-149,999$ & 247 & $(12.35)$ \\
for employment & 94 & $(4.70)$ & $150,000-199,999$ & 70 & $(3.50)$ \\
Other & 54 & $(2.70)$ & $200 \mathrm{k}+$ & 60 & $(03.00)$ \\
\hline
\end{tabular}

We also asked caregivers to report whether their care recipient had any chronic physical or mental health conditions. A large majority of care recipients had at least one chronic condition (92\%), and most had multiple chronic conditions. Specifically, $19 \%$ had one chronic condition, $25 \%$ had two chronic conditions, and $48 \%$ had three or more chronic conditions (median = two chronic conditions). Of those with chronic conditions, 33\% identified having at least one chronic mental condition (anxiety, depression, etc.), and $73 \%$ identified having at least one chronic physical condition (diabetes, high blood pressure, arthritis, etc.). High blood pressure was the most prevalent ( $42 \%$ of care recipients). Among chronic mental health conditions, $24 \%$ of care recipients have depression, with anxiety experienced by $23 \%$. Mental health conditions affected all age groups, while "physical" conditions affected care recipients increasingly more as they aged.

\subsection{Caregiver-Recipient Relationships}

The majority of caregivers in the sample were a family member of their care recipient (84\%). The two largest groups in the family member category were children $(41 \%)$ and spouses / significant
Table 4: Care recipients' type of healthcare insurance $(n=2000)$.

\begin{tabular}{c|r|r}
\hline Insurance & $<65 \mathrm{y} / \mathrm{o} \%$ & $\geq 65 \mathrm{y} / \mathrm{o} \%$ \\
\hline Medicaid & 39 & 13 \\
Medicare & 24 & 64 \\
Private Insurance & 17 & 14 \\
Veterans Health Care & 5 & 4 \\
\hline
\end{tabular}

Table 5: Respondents' self-reported type of residential community (by density) (left) and country region (right) $(n=2000)$.

\begin{tabular}{cc|cr}
\hline Density & $n(\%)$ & U.S. Region & $n(\%)$ \\
\hline Small town & & Midwest & $397(19.85)$ \\
or Rural & $638(31.9)$ & Northeast & $401(20.05)$ \\
Suburban & $978(48.9)$ & South & $799(39.95)$ \\
Urban & $384(19.2)$ & West & $403(20.15)$ \\
\hline
\end{tabular}


others $(20.4 \%)$ of the care recipient. These proportions are consistent with previous research $[10,46]$. The third largest group were not family members, but friends (11\%).

Almost half of caregivers lived with their recipient (43\%), and just over half lived apart (57\%). Of those who live apart from their caregivers, $77 \%$ lived within 30 minutes of their care recipient, and $83 \%$ reported visiting their care recipient in person at least once a week.

\subsection{Informal Caregiving Work}

The majority (56\%) of caregivers spent eight hours or more a week performing tasks related to caregiving. To narrow in on specific tasks associated with informal care work, we asked questions about activities the caregiver performs in support of another individual with a chronic condition or disability. Of more than 25 potential candidate tasks, caregivers selected activities that spanned three primary categories.

To arrive at these categories, the researchers considered Corbin and Strauss' Illness Trajectory Theory and lines of work: illness work, everyday life work, and biographical work (as mentioned in Section 3.3) [15]. We reviewed these lines of work alongside our caregiving research synthesis. Based on this review, we directly adopted Corbin and Stauss' notion of everyday life work, but expanded their concept of illness-related work to include medical and illness work, to surface increasing caregiver work with the healthcare system [24] while cultivating medical knowledge and managing biological illness trajectories. Finally, we saw a prominent role for psychosocial support [57] as its own line of work.

(1) Everyday living

Tasks in this category include grocery shopping (70\%), housework and home maintenance $(60 \%)$, providing transportation (59\%), and cooking or preparing meals (58\%).

(2) Psychosocial support

Tasks in this category include providing emotional support $(66 \%)$, visiting in-person regularly (54\%), and calling regularly $(50 \%)$.

(3) Medical and illness work

Medical work includes accompanying care recipients to medical appointments (63\%), managing and refilling medications (49\%), and coordinating with doctors, nurses, and medical providers (47\%). Illness work includes ADLs, such as personal care (bathing, dressing, etc.) (21\%), feeding (14\%), and toileting (11\%).

\subsection{Characteristics of Care Coordination}

When coordinating care between themselves and their care recipient, the primary communication method reported by caregivers was overwhelmingly phone calls $(71 \%)$. The next two most commonly cited communication methods were texting (33\%) and email (17\%). This overwhelming preference for communicating via phone calls was likely driven by the fact that many caregivers (43\%) reported that their care recipient did not own a smartphone. As a result, caregivers were less likely to use modes of communication that are reliant on smartphone use, such as text messaging, email, or messenger applications. Participants also reported a mismatch between the primary communication modes and devices between caregivers and their recipients. P1529 told us their biggest challenge is, "Not having the person I'm caring with in the loop (my mother). She's 90 and doesn't use ANY technology so when we all coordinate by email or text one of us has to call and fill her in."

As is evident in P1529's statement, coordinating care for many is not limited to coordination with their care recipient, but also other caregivers. The majority of caregivers reported coordinating care with at least one other person, not including professionals (59\%). When coordinating care with another caregiver, the primary methods of communication were phone calls $(69 \%)$ and text messaging (57\%), followed by email (27\%). Generally, caregivers were much more likely to use other methods of communication, in particular text messaging, when communicating with other caregivers compared to their care recipients.

When coordinating care with other caregivers, the majority do so at least once a week (72\%) and half reported coordinating several times a week or more (51\%). The most commonly cited reason for coordination was to check in with another caregiver to confirm a task has been done (50\%). Many also reported coordinating to share instructions with another caregiver (42\%) or to hand off responsibility to another person (to go out, work, sleep, etc.) (40\%).

\subsection{Challenges Coordinating Care: Primary Factors}

If a survey respondent identified that they coordinated care with another person in any way, we asked them, "What are your biggest challenges or difficulties in coordinating care with another person?" We received 1033 free entry responses to this question (57\% of respondents), which were the primary focus of our inductive, thematic analysis described in 3.3. Below, we report on findings from this analysis.

Through our thematic analysis, we generated two primary categories of themes: Coordination Challenges and Mediating Factors. Coordination challenges include three sub-components: Managing and Administrating, Communicating, and Scheduling. Both primary coordination challenges (3) and mediating factors (3) named at a high level are categories aimed to capture diversity of meaning in relation to a shared topic. Each domain category contains underlying themes.

Mediating factors were situational, and influenced the extent to which care coordination challenges were felt. Our findings suggest that mediating challenges regularly interact with one or more coordination challenges, such that they influence the degree of the challenge. They can extend beyond the control of the individual caregiver or recipient, to reflect broader biological, psychosocial, structural, or organizational phenomena that implicitly or explicitly influence which challenges arise and how (e.g., memory and cognitive limitations influencing medication schedules and communication about taking medication). These mediating factors also contained three sub-components: Relationships, Access to Resources, and Physical or Mental Barriers. Below, we introduce the findings relating to the three categories of themes describing primary coordination challenges, followed by findings on mediating factors. 
Table 6: Care Coordination Challenges: Overview of primary domain categories and their themes

\begin{tabular}{ll}
\hline Challenges & Themes \\
\hline Communicating & Communication response time \\
& Communication breakdowns \\
& Ensuring things are understood \\
& Keeping everyone up-to-date \\
& Disagreements with other caregivers \\
& Managing the entire caregiving process \\
Managing and administrating & Time management \\
& Task management, communication, and fairness \\
& Awareness of caregiver availability \\
Scheduling and temporal alignment & Reconciling caregiving schedules with work and life \\
& Dynamic changes \\
&
\end{tabular}

\subsection{Communicating}

Many caregivers described communication as their primary challenge when coordinating care. The specific challenges in this category included communication response time, communication breakdowns, ensuring information is understood, keeping all caregivers involved up-to-date, and disagreements. We elaborate on each of these challenges below.

4.6.1 Communication response time. Response time was the most common issue cited (46 instances) and often referred to delays between initiating and reaching someone the caregiver needed to connect with, and delays in communications of important information, generally between caregivers. Many of the open-ended responses included phrases such as "Getting in touch with them". As P757 told us, their biggest challenge is, "Getting in touch with [the person I coordinate with]. Sometimes they don't answer their phone when I need to tell them something important."

4.6.2 Communication breakdowns. Caregivers also expressed general communication breakdowns (43 instances). These issues generally described some difficulty with communication after-the-fact. Common words used included "miscommunication" or "misunderstood”. Participants noted a variety of breakdowns. For many, those with whom they coordinate can make mistakes based on misunderstandings. P1683 told us the person with whom they coordinate "misunderstood my message [and] told the wrong person the information." Still other respondents referred to breakdowns related to situational awareness that could impede knowing what topics to discuss. P1992 experiences challenges when communicating with other caregivers due to "not knowing what information to talk to them about."

4.6.3 Ensuring things are understood. A subset of communication challenges were closely related to managing and administrating care, in particular, issues with the process of how activities unfold. For example, caregivers mentioned coordination problems relating to making sure other caregivers know the plans or task details (27 instances). Common words included "making sure" or "remembering" in relation to communicating all the relevant information. For example, P1646 mentioned the worry they feel wondering if a message has been received, stating that when coordinating with others they "worry about whether they got the message." In addition to the delivery of information being received, caregivers need confirmation that instructions are followed. As P1991 told us, their challenge is "ensuring that all information is received and undertaken to the letter."

4.6.4 Keeping everyone up-to-date. A closely-related issue to making sure other caregivers know the plans or task details, and complete them, is the work involved in keeping all the caregivers up-todate / on the same page (19 instances). Almost all of the open-ended responses related to this challenge discussed some difficulty keeping people "on the same page." For example P280, listing several challenges, told us their biggest difficulty is, "Making sure we're on the same page, making sure there is constant coverage, making sure the other caregiver understands the focus of the visit."

4.6.5 Disagreements with other caregivers. Disagreements arose as a key communication challenge affecting coordination (13 instances). Examples of disagreement spanned differences in aspects of how people communicated (e.g., personal styles and modes of communication) as well as approaches different caregivers wanted to take to caregiving. For example P1736 told us they experience, "Differences in what we feel [the] patient needs." Disagreements can also arise when multiple caregivers interpret information or health status differently. For example, P60 wrote about the challenge of, "Siblings not agreeing on Mom's condition."

\subsection{Managing and Administrating}

Caregivers in our survey described a wide range of coordination challenges associated with managing the caregiving process and the tasks of others involved, as well as life outside their caregiving role. The activities in this category involved aspects of communication, information tracking and sharing, and time and task management in relation to other caregivers, the care recipient, and themselves.

4.7.1 Managing the entire caregiving process. Managing the totality of caregiving work was a key challenge for respondents (mentioned 23 times). Fourteen respondents commented on their own ability to 
keep track of, and remember things related to managing the caregiving process, for example "Remembering to discuss items" (P1190) and "Remembering to pass along all the information" (P1128). For other informal caregivers, managing the whole caregiving process requires being present or "being there" through different caregiving situations. For example, P455 described their primary challenge as, "being there at certain times to coordinate schedules, meeting in person and just being on top of everything." P944 stated their challenge as, "they can't do what needs to be done when I am not available." Still other caregivers commented on temporal factors more so than physical-namely, the difficulty of managing multiple things "at once." P1059 summed up this challenge, explaining, "Fust keeping everything organized and planning everything at once can be difficult."

4.7.2 Time management. Respondents also cited time management (mentioned 59 times) as their largest challenge coordinating care. Many open-ended responses simply expressed "time" as the largest challenge, but some participants offered richer descriptions. From these longer accounts of challenges, we learned that it is not just the time needed to administer or conduct care tasks that stood out but also the time it takes to check on tasks, prepare for administration of them, and communicate as part of care coordination. We heard, for example, that, "the time it takes to communicate and deciding who will do what" was P1734's biggest challenge.

4.7.3 Task management, communication, and fairness. Managing the tasks of other caregivers was the second most common set of challenges respondents mentioned. The most frequently-cited issue in this category was insuring other caregivers completed their tasks (mentioned 38 times). For example, P1854's largest challenge was, "Communication and the other care person following thru[sic] with the tasks." Assuring that other caregivers know what they are expected to do was mentioned by 27 respondents. Other challenges included the need to assure coverage for tasks-who is responsible for specific tasks (mentioned 15 times). As P249 explained, "Several caregivers are involved and they sometimes change their work schedules so I am not sure who will be giving the care when." Finally, the need for fair distribution of work among caregivers was mentioned six times as a primary challenge affecting coordination. P1386 wrote about the "Constant battle about who must do what" while P1342 commented on the importance of "Sharing responsibility so one person doesn't do all the work."

\subsection{Scheduling and Temporal Alignment Work}

Scheduling emerged as another key challenge in coordinating care. Participants reported issues with availability, coordinating schedules, working around scheduling constraints (e.g., jobs), and sudden changes to schedules. P1163 summarized the challenges in this category, explaining that, for them, "Who is to do what, when, and getting our calendars coordinated" is the largest challenge.

4.8.1 Awareness of caregiver availability. Survey respondents mentioned the need to maintain awareness of the overall availability of caregivers as the most frequent scheduling challenge (35 mentions). We learned that "Determining what times they will be available" was P950's greatest challenge. Participants reported difficulty with this task, as well as the need to find caregivers to cover specific tasks
Table 7: Mediating Factors: Overview of primary domain categories and their themes

\begin{tabular}{ll}
\hline Mediating Factors & Themes \\
\hline Relationships & $\begin{array}{l}\text { Trust and reliability } \\
\text { Introspection work } \\
\text { Care recipient autonomy }\end{array}$ \\
Access to Resources & $\begin{array}{l}\text { Physical proximity and social support } \\
\text { Financial and transportation resources }\end{array}$ \\
Barriers & $\begin{array}{l}\text { Physical } \\
\text { Mental }\end{array}$ \\
\hline
\end{tabular}

during specific days and times. We heard, for example, "the other person being able to work around the schedule I need" was P503's greatest challenge. This sentiment was echoed by P627, who mentioned, "Finding someone who's available at the time I need them" was challenging.

4.8.2 Reconciling caregiving schedules with work and life. The general act of coordinating schedules among caregivers was mentioned by 39 participants, while the specific task of reconciling caregiver schedules with work schedules was cited by 32 participants as their biggest challenge. Some participants reported feeling the burden of extra caregiving as a result of another caregiver's work obligations, while others specifically mentioned the challenge of accommodating another work schedule-especially when those schedules were changing. As P521 told us, "My alternate caregiver has a job so sometimes he is not available. I have to schedule my activities on his day off from work." Some respondents provided rich descriptions of how difficulty reconciling schedules and activities manifests in their lives. For example, P1768 told us, "My sister and I share responsibility for both of our parents in addition to having busy lives of our own. The most difficult thing is figuring out timing that allows us to accomplish everything and make sure that our parents are fed and medicated and entertained."

4.8.3 Dynamic changes. Finally, the need to respond to sudden changes to the caregiving or caregiver schedule was another top challenge, reported 19 times. Respondents' struggles included, "[c] hanges in the schedule of the person I care for or changes in either my or the other caregiver's schedule" (P681), and, "[i]f something comes up we have to reschedule everything around it" (P410).

\subsection{Challenges Coordinating Care: Mediating Factors}

In addition to the themes we introduce above describing primary challenges, we also identified three groups of mediating factors. These mediating factors effect the challenges mentioned above, bearing a direct influence on the extent to which caregivers experienced them. They include relationships, access to resources (including physical, financial, and social forms of support), and physical or mental barriers. Below, we elaborate on each category and the themes they comprise. 


\subsection{Relationships}

The largest group of mediating factors affecting care coordination concerned the interpersonal dimensions of the relationships caregivers have with their recipients, other caregivers, and themselves (186 mentions). Caregivers who are new to the care situation, care recipient, or other caregivers need time to cultivate the common ground that is needed to fully understand information and caregiving tasks. For example, P1763 told us, "If the other caregiver is new or unfamiliar, they may not understand everything you ask."

4.10.1 Trust and reliability. When coordinating care with other caregivers, the most common mediating factor cited was the reliability of the other caregiver (24 mentions). Caregivers generally described how other caregivers may fail to show up consistently, and the need to make sure tasks are completed by another caregiver correctly (or at all). For example, P1518 described their difficulty as, "Depending on [other caregiver [s] to show." This sentiment was echoed several times, such as in P1267's challenge, "Making sure they will do what they say and be dependable," and P1703 mentioning, "They can sometimes mess up something that you already had under control." In addition to the challenges our respondents' expressed trusting and relying on others, some also felt that others lacking trust in them was their primary challenge. As P129 explained, "I share care with a family member [and] the biggest challenge is getting things done my way, because she's related but not closer to him. I find she doesn't always want what I think is best because she is family."

4.10.2 Introspection work. The degree to which caregivers were able to do introspection work seemed to have an effect on their caregiving coordination. Some respondents reflected on their intrapersonal relationship, internal experiences acclimating to the realities of caregiving, and the declining health of their loved ones (23 mentions). This theme includes the caregivers' self concept, e.g., "II] don't want to think I can't do it all" (P1156). It also comprises emotion management, and difficulty achieving peace of mind about the state of the care recipient, e.g., "Knowing how they're feeling in these moments" (P1868). Finally, some responses eluded to the personal difficulty of coming to terms with lack of control over circumstances, as P131 stated, "No matter what I do things will just get worse as time goes on."

4.10.3 Care recipient autonomy. The degree to which the autonomy of the recipient and the locus of control of the situation can be negotiated had an affect on caregivers' challenges (17 mentions). There is natural tension between the needs of the caregiver to provide care (e.g., conduct tasks on the care recipient's behalf, give instructions, orchestrate activities) and the desire of the care recipient to maintain independence. We heard from P1218, "My mother likes to give instructions. She does not like to receive instructions, for example, instructions with medications." For some respondents, this manifested as negotiation of the locus of control of situations. P663 described their challenge as, "The care recipient accepting the care as arranged and not trying to be in charge."

\subsection{Access to Resources}

Another mediating factor pertained to access to, or lack of access to, resources (117 mentions). The degree to which respondents could access resources affected their physical circumstances, life circumstances, and both the caregivers' and the care recipients' health status.

4.11.1 Physical proximity and social support. The most common issue cited in this category was distance from the recipient (38 instances). This included physical distance between the caregiver and their recipient, and also problems of distance and coordinating care between other caregivers. For example, P1280 spoke of the challenge, "Living so far away and having my own family to take care of."

Respondents also cited a lack of support from other caregivers (12 instances). This suggested that the majority of caregiving responsibilities fell onto themselves, or they lacked others upon whom they could rely. For example, P48 mentioned, "Not having another person I can count on." This sentiment was echoed by P1845, who noted, "Lack of support. Doing it all myself," and P371, who expressed their concern that, "There is no one else if something happens to me." Responses also suggested that caregivers experienced their own lack of autonomy without support. For example, P223 mentioned having "no freedom" while P1621 mentioned the challenge of finding "Time for myself to be off."

4.11.2 Financial and transportation resources. Caregivers' financial resources and access to transportation also mediated their ability to meet caregiving challenges. Several respondents mentioned "money" as their primary challenge (4 instances). For example, P1088 told us their primary challenge was, "having enough money to pay for a caregiver." $\mathrm{P} 752$ reported their challenge as, "The non ending continue [d] care with no pay." Transportation issues were mentioned by six respondents as their biggest challenge coordinating care.

\subsection{Physical and Mental Barriers}

Forty-seven caregivers reported physical or mental mediating factors affecting their care coordination. These factors could affect the care recipient, other caregivers, or the respondent themselves. One of the most common issues cited pertained to physical limitations of others (12 mentions). These barriers tended to be primarily related to the physical limitations of care recipients (8 mentions), and, to a lesser extent, the limitations of other caregivers (4 mentions). For example, P616 mentioned struggling with their care recipient's, "mobility and ease of walking" while P274's largest challenge was their recipient's "hearing problems." These physical challenges influence how care is coordinated and administered, as P1872 illustrated, "[...] the bathroom care is most difficult."

Nine respondents mentioned memory issues as a factor affecting their ability to coordinate care. It was not always clear whether the respondent was referring to the memory of the recipient or of another caregiver. However, common problems cited involved both specific tasks, such as forgetting what was said, and general difficulties with memory loss, such as the recipient suffering from dementia-related memory loss. For example, P1863 mentioned, "When they forget to do something like taking their medication." Speaking more generally, P744 mentioned, "They forget what was said or done," while P3 mentioned, "Battling their memory problems...dementia."

Finally, six respondents described physical and mental mediating factors solely reflecting the personal health toll of caregiving. The 
most common phrases were centered around exhaustion or fatigue. P1655 reported that their biggest challenge coordinating care is that it, "Can be very emotionally tiring. Can be physically tiring. Finding time to do all of it." P1297 mentioned the challenge of, "Having enough energy yourself."

\section{DISCUSSION}

Our study findings contribute to the large body of research illustrating the many forms of informal caregiving work and caregivers' coordination challenges. Below, we reflect on our findings and situate them with respect to prior work. We then discuss implications and opportunities we see for research and design to better support caregivers. We conclude with a discussion of limitations of our study and directions for future work.

\subsection{Reflections on findings}

Although we expected that the modality of the study, being an online survey, might elicit shallow statements about more tactical aspects of completing care tasks, we found that respondents cited coordination challenges spanning complex inter- and intrapersonal experiences. Our findings, taken together with prior work, suggest that caregivers bring physical, mental, and social-emotional effort into the coordination of everyday life work, medical and illness work, and psychosocial support they provide. This work is mediated by factors that often extend beyond the individual caregiver, such as their relationships to others. For example, trust and reliability (which are integral to relationships between caregivers) have mediating effects on how disagreements about care tasks are resolved (a key challenge for caregivers in our survey).

Our survey findings suggest that several situational, mediating factors influence the extent to which care coordination challenges were felt. These factors include relationships (as discussed above) and also, access to resources, and physical and mental barriers. These mediating factors:

- Regularly interact with one or more coordination challenges such that they influence the degree of the challenge.

- Often extend beyond the control of the individual caregiver or recipient, to reflect broader biological, psychosocial, structural, or organizational phenomena that implicitly or explicitly influence which challenges arise and how (e.g., memory and cognitive limitations can influence adherence to a medication schedule and communication about taking medication).

5.1.1 How our findings compare to prior work. Our findings on primary coordination challenges echo and support results of prior work. Tang et al. found three main challenges in the coordination of care across multiple caregivers. First, caregivers had difficulty maintaining awareness of patient information among caregivers. We saw this in our data as well, emerging in statements about challenges keeping everyone up-to-date or on the "same page".

Our survey findings suggest that coordination work to keep everyone on the "same page" is impacted by breakdowns, including gaps in hand-offs among caregivers [65] that, in turn, impact illness and everyday life work [6], and gaps in situational awareness [20] needed for effective work as a caregiver [29]. Work to create technology to support aspects of situational awareness [74] has begun to set the direction for possibilities in this space.

We also know from prior work that enabling caregivers to repair communication breakdowns is important for interpersonal reasons and relation work, such as easing family conflict [6, 25, 75], easing tensions, and building trust $[16,59]$. Breakdowns impact both caregivers (e.g., who often work to repair a breakdown) as well as the recipient, about whom breakdowns occur (e.g., affecting both care tasks and relationships).

Revisiting our findings related to hand-offs, our survey found hand-off activities to be prominently important, echoing many prior studies $[6,12,25,75]$. Like the "gaps in patient handoffs" Tang et al. [65] described, our survey respondents referred to similar challenges, such as insuring other caregivers completed their tasks, coordinating schedules among caregivers, and responding to sudden changes to the caregiving or caregiver's schedule. Indeed, the most commonly-cited reason for coordination was to check in with another caregiver to confirm a task has been done, followed by the need to share instructions with another caregiver, and finally, to hand off the responsibility of a task to another person.

Finally, prior work highlights relationship tensions inherent in caregiving [6, 65]. We saw these difficulties emerge in statements about disagreements with other caregivers, fair distribution of work among caregivers, and the need to support both care work and care recipient autonomy.

\subsection{Design implications and opportunities for HCI to support caregivers}

5.2.1 Supporting interpersonal relation work. While respondents indeed mentioned the need for support with "tasks" (e.g., help scheduling other caregivers' time), just as common were challenges that transcend tasks and prescribed activities, and suggest difficult internal and interpersonal relation work. This is consistent with what we found through our synthesis of research on informal caregiving: interpersonal work plays a large role in coordination and intersects with articulation and information work. Given the evidence we see on the importance of human relationships in mediating care coordination challenges, designing technology and sociotechnical systems not for the "user" but for the interpersonal dynamics of their "relationships" should guide design processes and evaluation outcomes. These challenges point to new areas for $\mathrm{HCI}$ inquiry. For example:

Many informal caregivers are reluctant or simply unable to express challenges or recount their labor, which can, in turn, lead to a misrepresentation and lack of awareness of opportunities for support and collaboration $[12,25]$.

How can we look at informal caregiving in workercentered [22] ways, and create visibility and social acknowledgement of what is currently invisible work [62] of caregivers?

Questions of how sociotechnical systems might support acknowledgment of work will be non-trivial to answer, as visibility of work has trade-offs: it "can create reification of work, opportunities for surveillance, or come to increase group communication and process 
burdens" [62, 64]. Indeed, the visibility-invisibility tension of work surfaced as accounts of disagreement in our survey, such as P154's statement, "Sometimes we are doing things differently and they think their way is the only right way," and P129's comment that "getting things done my way" is the hardest challenge. These sentiments suggest that more visibility into individual caregivers' work could surface tensions, rather than lead to the kinds of acknowledgment that are currently missing. Thus, we must consider approaches to visibility that support caregivers' broader need for social support [57].

5.2.2 Supporting introspection work. Our qualitative analysis focused on responses to the question: "What are your biggest challenges or difficulties in coordinating care with another person?" Yet, many respondents (23) commented in ways that suggested difficulties managing introspection work that, in turn, affected their care coordination with others. For example, P1156's comment, "I don't want to think I can't do it all," suggests an important role for the intrapersonal work of self-concept development and identity adaptation.

Indeed, prior work has found that both caregivers and care recipients must acclimate to new roles and shifts in identity. For example, people with chronic conditions interpret their situations and their health status by looking for indicators of their ability to accomplish daily pursuits, which can change over time and which act as "markers" of the self [11]. For those who care for others, daily pursuits of the caregiver and care recipient are interwoven: the caregiver supports the recipient in achieving everyday tasks that are integral to their daily life. Thus, it can be difficult for the caregiver to prioritize both their own pursuits (e.g., social activities, fitness) and the needs of the care recipient (e.g., activities of daily living). As prior habitual patterns of the informal caregiver gradually change to accommodate those in their care, they risk the loss of aspects of their own identity [26]. As such, a related prompt for the HCI community is:

How can we understand and design for the role of technology in supporting introspection work for the caregiver and recipient, as they negotiate new roles, dependencies, self concepts, and shifting abilities?

5.2.3 Getting acclimated to caregiving and caregiver relationships. Our findings support the need for design implications outlined in previous work: systems that are designed with a focus on the clinician and patient do not reflect the reality of the patient's ecosystem of care [12, 13, 23, 24, 39]. Instead, sociotechnical care systems must be designed for clinicians, care recipients (i.e., patients), and a variety of informal caregivers that provide day-to-day care outside of a clinical setting. We expand on previous work by presenting design opportunities that can help the main caregiver, integrate secondary caregivers, and maintain the care recipient as an active participant in their own care.

Traditionally, sociotechnical systems have focused on the design of productivity tools (e.g., calendaring, record-keeping apps, and integration of communication channels) and "tracking" to address the challenges of coordination and collaboration in caregiving contexts. Our work surfaces new opportunities for sociotechnical systems of care. In particular:
How can new caregivers be supported in getting acclimated to life as a caregiver, through systems that provide accessible knowledge and advice?

How can systems scaffold the process of task management among caregivers, such that knowledge, caregiving experience, and level of engagement is taken into account when distributing caregiving tasks?

How can scaffolding approaches be used to enable trust and foster a sense of connectedness among caregivers and the care recipient?

Use of scaffolding in HCI builds on work in the learning sciences [17], on how people advance in their learning from tasks that they can do already (without help) to those that they can achieve with the right level of assistance (or scaffolds) [55]. In HCI, the concept of scaffolding has been used in many application areas and refers to both human and computational scaffolds. Prior work has explored scaffolding approaches in helping parents and their children develop teacher-recommended socioemotional skills [60], in interactive learning environments [49, 72], in VR games in which people must learn interactions [71], to support novice programmers to complete software development tasks [70], and to scaffold design work [17, 28] (to name a few areas). We see opportunity to explore scaffolding much more in the context of collaborative caregiving, building on work that finds that scaffolding approaches can assist people to acquire and develop reflective thinking skills in condition selfmanagement [36].

Designing for scaffolding and trust in the context of caregiving presents opportunities and challenges, but doing so has the potential to alleviate the burden on the primary informal caregiver, by enabling them to receive assistance from other informal caregivers, even if they have less experience. As new caregivers gain confidence and knowledge by participating in caregiving tasks, additional tasks can increase in complexity and involvement. Last, including the care recipient as part of the team could ensure that they maintain agency (when possible) by participating in caregiving tasks and being connected to the day-to-day care activities in which the primary and secondary caregivers take part.

5.2.4 Algorithmic coordination and human expertise. As computing systems advance to include more and more automation and AI-driven assistance, it is likely that computing researchers and companies will propose "solutions" for care coordination that include algorithms for distributing tasks, assigning activities to people in a caregiving network, and enabling tracking of caregiving activities and status. As such, important questions arise related to fairness in task distribution, privacy and surveillance, and the need to balance system recommendations with human autonomy. It is vital that informal caregivers have ongoing input into the design of any systems that enable algorithmic and/or clinical decision-making [20]. This is important both to ensure that caregivers participate in the design process end-to-end, but also to ensure that, once technology is deployed, it is beneficial, rather than disruptive, to caregiving communities. As such, prompts for the HCI community include:

How can designers and developers of AI assistant technology ensure that caregivers and recipients maintain the locus of control and agency they need in planning, 
coordinating, and conducting care-related activities, once AI is introduced?

How can we ensure that the well-being of caregivers and care recipients is a priority objective for any computing system that we develop and introduce into caregiving contexts?

5.2.5 The importance of access to resources. Respondents' descriptions of resource constraints and access challenges point to complex questions about the role of sociotechnical systems in addressing such challenges. One active area of work includes research on the types of work caregivers conduct to navigate healthcare resource constraints, including financial constraints. Gui et al. discussed the "strategic infrastructuring work" caregivers did to work around these constraints [24]. Looking at factors such as access to financial resources as an influence on care coordination work could also help shift our analytic and design lenses toward meso- and macro-level phenomena that have systemic, mediating influences on specific caregiving challenges.

Considering that access to resources is a mediating factor influencing caregivers' coordination challenges, it is vital to acknowledge that technology interventions that support health management can actually exacerbate health disparities by disproportionately benefiting more advantaged people [68].

As more and more technologies emerge to support care management at home, how can we make sure that solutions we propose would not exacerbate the already-prevalent resource challenges of caregivers?

How do we prevent the possibility of increasing disparities between those who can access the technologies and services needed for our solutions to work, and those who cannot? How can we instead work toward reducing such disparities?

\subsection{Limitations and Future Work}

Although we sampled a large swath of the U.S. population, our findings are meant to be descriptive. As such, further research is required to determine whether subgroup analyses could be conducted with enough power to provide reliable inferential insights about specific caregiver groups.

While our survey elicited caregivers' perspectives on their most salient challenges coordinating care, the nature of the survey method, while powerful in its reach, is limited in its ability to elicit context, reflection, and deeper narrative. Future work could complement our large-scale data analysis with deeper qualitative inquiries into the challenges and mediating factors that we surfaced in findings and discussion. Interview studies in the areas outlined in our discussion, for example, could provide a vital complement to our survey method. In particular, as research pursues more of the intrapersonal challenges of caregiving-a currently under-explored area-interviews could support evocative engagements with multiple participants in the care coordination network to elicit deeper reflection and narratives of caregivers' lived experiences [54].

Our survey was designed and deployed in mid-2019, and data analysis spanned 2019-2020. The global pandemic has since transformed how medical care and other services and goods are accessed.
It will be important to repeat this study to understand how care coordination and other care work shifts to respond to the need to inhabit virtual settings (e.g., online doctor appointments) or the need to interleave virtual and physical tasks (e.g., ordering supplies or goods online to avoid human contact). Findings from this survey might serve as a "pre-pandemic" basis for comparison with future findings.

While we give counts of the occurrences of the themes mentioned in our data, we note that higher frequency of a stated challenge does not increase the importance of solving that challenge over others. We note that there is a tendency of survey respondents to answer questions in a manner that will be viewed favorably by others-or "social desirability bias" [33]. Responses that might suggest difficult circumstances or an undesirable social situation of the respondent could be suppressed subject to this bias.

Finally, while $59 \%$ of our survey respondents reported that they coordinate care with at least one other person, only about $57 \%$ offered a response to the open-ended question about care coordination challenges. We cannot know why those who did not answer the open-ended question chose not to respond. It is possible that no challenges came to mind, or that the challenges they experience were considered to too personal, or even too lengthy, to describe in a survey response.

\section{CONCLUSION}

We present findings of a large survey examining the care work and care coordination challenges of 2000 informal caregivers in the USA. Findings from the study serve as a descriptive complement to qualitative studies, and concur with findings of prior research on the specific coordination challenges of informal caregiver groups: communication challenges, managing and administering the wide range of needed care activities, and scheduling and temporal alignment tasks. Our study also offers insights into mediating factors that influence the degree to which these challenges are felt. By illuminating how primary coordination challenges and mediating factors affect a large set of caregivers, we can better inform the design of sociotechnical systems to support informal care work.

\section{ACKNOWLEDGMENTS}

We would like to acknowledge Dr. Michael Howell, and all survey respondents who took the time to share their caregiving perspectives.

\section{REFERENCES}

[1] Rikke Aarhus, Erik Grönvall, and Morten Kyng. 2010. Challenges in participation. In 2010 4th International Conference on Pervasive Computing Technologies for Healthcare. IEEE, 1-4.

[2] Ofra Amir, Barbara J. Grosz, Krzysztof Z. Gajos, Sonja M. Swenson, and Lee M. Sanders. 2015. From Care Plans to Care Coordination: Opportunities for Computer Support of Teamwork in Complex Healthcare. In Proceedings of the 33rd Annual ACM Conference on Human Factors in Computing Systems (Seoul, Republic of Korea) (CHI '15). Association for Computing Machinery, New York, NY, USA, 1419-1428. https://doi.org/10.1145/2702123.2702320

[3] Stinne Aaløkke Ballegaard, Jonathan Bunde-Pedersen, and Jakob E Bardram. 2006. Where to, Roberta? Reflecting on the role of technology in assisted living. In Proceedings of the 4th Nordic conference on Human-computer interaction: changing roles. 373-376.

[4] The World Bank. 2019. United Nations Population Division's World Population Prospects: 2019 Revision. https://data.worldbank.org/indicator/SP.POP.65UP. TO.ZS 
[5] Andrew B L Berry, Catherine Lim, Andrea L Hartzler, Tad Hirsch, Evette Ludman, Edward H Wagner, and James D Ralston. 2017. Creating Conditions for Patients' Values to Emerge in Clinical Conversations: Perspectives of Health Care Team Members. In Proceedings of the 2017 Conference on Designing Interactive Systems (DIS '17). ACM, New York, NY, USA, 1165-1174. https://doi.org/10.1145/3064663. 3064669

[6] Tone Bratteteig and Ingvild Eide. 2017. Becoming a good homecare practitioner integrating many kinds of work. Computer Supported Cooperative Work (CSCW) 26, 4-6 (2017), 563-596.

[7] Virginia Braun and Victoria Clarke. 2019. Reflecting on reflexive thematic analysis. Qualitative Research in Sport, Exercise and Health 11, 4 (2019), 589-597.

[8] Virginia Braun and Victoria Clarke. 2020. One size fits all? What counts as quality practice in (reflexive) thematic analysis? Qualitative research in psychology (2020), $1-25$.

[9] US Census Bureau. 2010. US Census Regions. https://www2.census.gov/geo/ pdfs/maps-data/maps/reference/us_regdiv.pdf

[10] AARP Family Caregiving and National Alliance for Caregiving. 2020. Caregiving in the U.S. https://www.aarp.org/content/dam/aarp/ppi/2020/05/full-reportcaregiving-in-the-united-states.doi.10.26419-2Fppi.00103.001.pdf

[11] Kathy Charmaz. 2006. Measuring pursuits, marking self: Meaning construction in chronic illness. International fournal of Qualitative Studies on Health and Well-being 1, 1 (2006), 27-37.

[12] Yunan Chen, Victor Ngo, and Sun Young Park. 2013. Caring for Caregivers: Designing for Integrality. In Proceedings of the 2013 conference on Computer Supported Cooperative Work. ACM, 91-102.

[13] Yunan Chen, Nervo Verdezoto, Xinning Gui, Xiaojuan Ma, Claus Bossen, Naveen Bagalkot, Valeria Herskovic, and Bernd Ploderer. 2019. Unpacking the Infrastructuring Work of Patients and Caregivers Around the World. In Extended Abstracts of the 2019 CHI Conference on Human Factors in Computing Systems (Glasgow, Scotland Uk) (CHI EA '19). ACM, New York, NY, USA, Article W03, 8 pages. https://doi.org/10.1145/3290607.3299021

[14] Sunny Consolvo, Peter Roessler, Brett E Shelton, Anthony LaMarca, Bill Schilit, and Sara Bly. 2004. Technology for care networks of elders. IEEE pervasive computing 3, 2 (2004), 22-29.

[15] Juliet Corbin and Anselm Strauss. 1985. Managing chronic illness at home: three lines of work. Qualitative sociology 8, 3 (1985), 224-247.

[16] Flannery Currin, Gustavo Razo, and Aehong Min. 2019. Give Me a Break: Design for Communication Among Family Caregivers and Respite Caregivers. In Extended Abstracts of the 2019 CHI Conference on Human Factors in Computing Systems (Glasgow, Scotland Uk) (CHI EA '19). ACM, New York, NY, USA, Article SDC05, 6 pages. https://doi.org/10.1145/3290607.3309687

[17] Betsy DiSalvo. 2016. Participatory Design through a Learning Science Lens. In Proceedings of the 2016 CHI Conference on Human Factors in Computing Systems (San Jose, California, USA) (CHI '16). Association for Computing Machinery, New York, NY, USA, 4459-4463. https://doi.org/10.1145/2858036.2858405

[18] Karen Donelan, Craig Hill, Catherine Hoffman, Kimberly Scoles, Penny Feldman, Carol Levine, and David Gould. 2002. Challenged To Care: Informal Caregivers In A Changing Health System. Health affairs (Project Hope) 21 (07 2002), 222-31. https://doi.org/10.1377/hlthaff.21.4.222

[19] Hayley Evans, Udaya Lakshmi, Hue Watson, Azra Ismail, Andrew M. Sherrill, Neha Kumar, and Rosa I. Arriaga. 2020. Understanding the Care Ecologies of Veterans with PTSD. In Proceedings of the 2020 CHI Conference on Human Factors in Computing Systems (Honolulu, HI, USA) (CHI '20). Association for Computing Machinery, New York, NY, USA, 1-15. https://doi.org/10.1145/3313831.3376170

[20] Pin Sym Foong, Charis Anne Lim, Joshua Wong, Chang Siang Lim, Simon Tangi Perrault, and Gerald CH Koh. 2020. " You Cannot Offer Such a Suggestion" Designing for Family Caregiver Input in Home Care Systems. In Proceedings of the 2020 CHI Conference on Human Factors in Computing Systems. 1-13.

[21] Pin Sym Foong, Shengdong Zhao, Felicia Tan, and Joseph Jay Williams. 2018 Harvesting Caregiving Knowledge: Design Considerations for Integrating Volunteer Input in Dementia Care. In Proceedings of the 2018 CHI Conference on Human Factors in Computing Systems (Montreal QC, Canada) (CHI '18). ACM, New York, NY, USA, Article 79, 12 pages. https://doi.org/10.1145/3173574.3173653

[22] Sarah E Fox, Vera Khovanskaya, Clara Crivellaro, Niloufar Salehi, Lynn Dombrowski, Chinmay Kulkarni, Lilly Irani, and Jodi Forlizzi. 2020. Worker-Centered Design: Expanding HCI Methods for Supporting Labor. In Extended Abstracts of the 2020 CHI Conference on Human Factors in Computing Systems. 1-8.

[23] Amir Goren, William Montgomery, Kristin Kahle-Wrobleski, Tomomi Nakamura, and Kaname Ueda. 2016. Impact of caring for persons with Alzheimer's disease or dementia on caregivers' health outcomes: findings from a community based survey in Japan. BMC geriatrics 16, 1 (2016), 122.

[24] Xinning Gui and Yunan Chen. 2019. Making Healthcare Infrastructure Work: Unpacking the Infrastructuring Work of Individuals. In Proceedings of the 2019 CHI Conference on Human Factors in Computing Systems. ACM, 458.

[25] Francisco J Gutierrez and Sergio F Ochoa. 2017. It takes at least two to tango: understanding the cooperative nature of elderly caregiving in Latin America. In Proceedings of the 2017 ACM Conference on Computer Supported Cooperative Work and Social Computing. ACM, 1618-1630.
[26] Betty R Hasselkus and Bridget J Murray. 2007. Everyday occupation, well-being, and identity: The experience of caregivers in families with dementia. American fournal of Occupational Therapy 61, 1 (2007), 9-20.

[27] Matthew K. Hong, Udaya Lakshmi, Kimberly Do, Sampath Prahalad, Thomas Olson, Rosa I. Arriaga, and Lauren Wilcox. 2020. Using Diaries to Probe the Illness Experiences of Adolescent Patients and Parental Caregivers. In Proceedings of the 2020 CHI Conference on Human Factors in Computing Systems (Honolulu, HI, USA) (CHI '20). Association for Computing Machinery, New York, NY, USA, 1-16. https://doi.org/10.1145/3313831.3376426

[28] Matthew K. Hong, Udaya Lakshmi, Thomas A. Olson, and Lauren Wilcox. 2018. Visual ODLs: Co-Designing Patient-Generated Observations of Daily Living to Support Data-Driven Conversations in Pediatric Care. In Proceedings of the 2018 CHI Conference on Human Factors in Computing Systems - CHI '18. ACM Press, New York, New York, USA, 1-13. https://doi.org/10.1145/3173574.3174050

[29] Matthew K Hong, Lauren Wilcox, Daniel Machado, Thomas A Olson, and Stephen F Simoneaux. 2016. Care Partnerships: Toward Technology to Support Teens' Participation in Their Health Care. In Proceedings of the 34th annual ACM Conference on Human Factors in Computing Systems. San Jose, CA. https://doi.org/10.1145/2858036.2858508

[30] AARP Public Policy Institute. 2017. Who Relies on Medicare? A Profile of the Medicare Population. https://www.aarp.org/content/dam/aarp/ppi/2017/11/whorelies-on-medicare-a-profile-of-the-medicare-population.pdf

[31] Maia Jacobs, Galina Gheihman, Krzysztof Z. Gajos, and Anoopum S. Gupta. 2019. "I Think We Know More than Our Doctors": How Primary Caregivers Manage Care Teams with Limited Disease-Related Expertise. Proc. ACM Hum.-Comput. Interact. 3, CSCW, Article 159 (Nov. 2019), 22 pages. https://doi.org/10.1145/ 3359261

[32] Elizabeth Kaziunas, Mark S. Ackerman, Silvia Lindtner, and Joyce M. Lee. 2017. Caring through Data: Attending to the Social and Emotional Experiences of Health Datafication. In Proceedings of the 2017 ACM Conference on Computer Supported Cooperative Work and Social Computing - CSCW'17. ACM Press, New York, New York, USA, 2260-2272. https://doi.org/10.1145/2998181.2998303

[33] Ivar Krumpal. 2013. Determinants of social desirability bias in sensitive surveys: a literature review. Quality \& Quantity 47, 4 (2013), 2025-2047.

[34] Ariella Lang. 2010. There's no place like home: research, practice and policy perspectives regarding safety in homecare.

[35] Leslie S Liu, Sen H Hirano, Monica Tentori, Karen G Cheng, Sheba George, Sun Young Park, and Gillian R Hayes. 2011. Improving communication and social support for caregivers of high-risk infants through mobile technologies. In Proceedings of the ACM 2011 conference on Computer supported cooperative work. 475-484.

[36] Lena Mamykina, Elizabeth Mynatt, Patricia Davidson, and Daniel Greenblatt. 2008. MAHI: Investigation of Social Scaffolding for Reflective Thinking in Diabetes Management. In Proceedings of the SIGCHI Conference on Human Factors in Computing Systems (Florence, Italy) (CHI '08). Association for Computing Machinery, New York, NY, USA, 477-486. https://doi.org/10.1145/1357054.1357131

[37] Medicaid.gov. 2020. Medicaid Statistics. https://www.medicaid.gov/medicaid/ index.html

[38] Medicaid.gov. 2020. What's Medicare? https://www.medicare.gov/whatmedicare-covers/your-medicare-coverage-choices/whats-medicare

[39] Andrew D Miller, Sonali R Mishra, Logan Kendall, Shefali Haldar, Ari H Pollack, and Wanda Pratt. 2016. Partners in care: design considerations for caregivers and patients during a hospital stay. In Proceedings of the 19th ACM Conference on Computer-Supported Cooperative Work \& Social Computing. ACM, 756-769.

[40] Pedro A Moreno, Saisakul Chernbumroong, Caroline Langensiepen, Ahmad Lotfi, and Enrique J Gómez. 2016. Virtual Carer: Personalized support for informal caregivers of elderly. In Proceedings of the 9th ACM International Conference on PErvasive Technologies Related to Assistive Environments. ACM, 38.

[41] Ingrid Mulder, Yvonne Schikhof, Martijn Vastenburg, Alan Card, Tory Dunn, Andreas Komninos, Marilyn McGee-Lennon, Mark Santcroos, Gabriele Tiotto, Mieke Van Gils, et al. 2009. Designing with care: The future of pervasive healthcare. IEEE pervasive computing 8, 4 (2009), 85-88.

[42] Elizabeth D. Mynatt, Jim Rowan, Sarah Craighill, and Annie Jacobs. 2001. Digital Family Portraits: Supporting Peace of Mind for Extended Family Members. In Proceedings of the SIGCHI Conference on Human Factors in Computing Systems (Seattle, Washington, USA) (CHI '01). ACM, New York, NY, USA, 333-340. https: //doi.org/10.1145/365024.365126

[43] Francisco Nunes, Nervo Verdezoto, Geraldine Fitzpatrick, Morten Kyng, Erik Grönvall, and Cristiano Storni. 2015. Self-Care Technologies in HCI: Trends, Tensions, and Opportunities. ACM Trans. Comput.-Hum. Interact. 22, 6, Article 33 (Dec. 2015), 45 pages. https://doi.org/10.1145/2803173

[44] International Alliance of Carer Organizations. [n.d.]. Carer Facts: Recognizing Carers. https://internationalcarers.org/carer-facts/

[45] US Dept of Veteran Affairs. 2020. About VHA. https://www.va.gov/health/ aboutvha.asp

[46] National Research Council (US) Committee on the Role of Human Factors in Home Health Care. 2010. Informal Caregivers in the United States: Prevalence, Caregiver Characteristics, and Ability to Provide Care. https://www.ncbi.nlm. 
nih.gov/books/NBK210048/

[47] Anthony J Onwuegbuzie and Kathleen MT Collins. 2007. A typology of mixed methods sampling designs in social science research. Qualitative Report 12, 2 (2007), 281-316.

[48] World Health Organization. 2020. Nutrition Topics. https://www.who.int/ nutrition/topics/2_background/en/

[49] Eleanor O’Rourke, Erik Andersen, Sumit Gulwani, and Zoran Popović. 2015. A Framework for Automatically Generating Interactive Instructional Scaffolding. In Proceedings of the 33rd Annual ACM Conference on Human Factors in Computing Systems (Seoul, Republic of Korea) (CHI '15). Association for Computing Machinery, New York, NY, USA, 1545-1554. https://doi.org/10.1145/2702123.2702580

[50] Leysia Palen and Stinne Aaløkke. 2006. Of pill boxes and piano benches: " home-made" methods for managing medication. In Proceedings of the 200620 th anniversary conference on Computer supported cooperative work. 79-88.

[51] Laura Pina, Sang-Wha Sien, Clarissa Song, Teresa M. Ward, James Fogarty, Sean A. Munson, and Julie A. Kientz. 2020. DreamCatcher: Exploring How Parents and School-Age Children Can Track and Review Sleep Information Together Proc. ACM Hum.-Comput. Interact. 4, CSCW1, Article 070 (May 2020), 25 pages. https://doi.org/10.1145/3392882

[52] Laura R. Pina, Sang-Wha Sien, Teresa Ward, Jason C. Yip, Sean A. Munson, James Fogarty, and Julie A. Kientz. 2017. From Personal Informatics to Family Informatics: Understanding Family Practices around Health Monitoring. In Proceedings of the 2017 ACM Conference on Computer Supported Cooperative Work and Social Computing (Portland, Oregon, USA) (CSCW'17). Association for Computing Machinery, New York, NY, USA, 2300-2315. https://doi.org/10.1145/2998181.2998362

[53] Anne Marie Piper, Raymundo Cornejo, Lisa Hurwitz, and Caitlin Unumb. 2016. Technological caregiving: Supporting online activity for adults with cognitive impairments. In Proceedings of the 2016 CHI Conference on Human Factors in Computing Systems. 5311-5323.

[54] Mirjana Prpa, Sarah Fdili-Alaoui, Thecla Schiphorst, and Philippe Pasquier. 2020. Articulating Experience: Reflections from Experts Applying MicroPhenomenology to Design Research in HCI. In Proceedings of the $2020 \mathrm{CHI}$ Conference on Human Factors in Computing Systems. 1-14.

[55] Debra Sanders and Dorette Sugg Welk. 2005. Strategies to scaffold student learning: Applying Vygotsky's zone of proximal development. Nurse educator 30, 5 (2005), 203-207.

[56] Kjeld Schmidt and Carla Simonee. 1996. Coordination mechanisms: Towards a conceptual foundation of CSCW systems design. Computer Supported Cooperative Work (CSCW) 5, 2-3 (1996), 155-200.

[57] Marén Schorch, Lin Wan, David William Randall, and Volker Wulf. 2016. Designing for those who are overlooked: Insider perspectives on care practices and cooperative work of elderly informal caregivers. In Proceedings of the 19th ACM Conference on Computer-Supported Cooperative Work \& Social Computing. ACM, 787-799.

[58] Carla Simone, Monica Divitini, and Kjeld Schmidt. 1995. A notation for malleable and interoperable coordination mechanisms for CSCW systems. In Proceedings of conference on Organizational computing systems. 44-54.

[59] Portia E. Singh, Payaal Patel, Matthew L. Lee, and Mladen Milosevic. 2016. Understanding Informal Caregiver Needs for Coordinating Care to Support Aging in Place. In Proceedings of the 10th EAI International Conference on Pervasive Computing Technologies for Healthcare (Cancun, Mexico) (PervasiveHealth '16). ICST (Institute for Computer Sciences, Social-Informatics and Telecommunications Engineering), Brussels, BEL, 249-252.

[60] Petr Slovák, Kael Rowan, Christopher Frauenberger, Ran Gilad-Bachrach, Mia Doces, Brian Smith, Rachel Kamb, and Geraldine Fitzpatrick. 2016. Scaffolding the Scaffolding: Supporting Children's Social-Emotional Learning at Home. In Proceedings of the 19th ACM Conference on Computer-Supported Cooperative Work \& Social Computing (San Francisco, California, USA) (CSCW '16). Association for Computing Machinery, New York, NY, USA, 1751-1765. https://doi.org/10.1145/
2818048.2820007

[61] Katta Spiel, Oliver L. Haimson, and Danielle Lottridge. 2019. How to Do Better with Gender on Surveys: A Guide for HCI Researchers. Interactions 26, 4 (June 2019), 62-65. https://doi.org/10.1145/3338283

[62] Susan Leigh Star and Anselm Strauss. 1999. Layers of silence, arenas of voice: The ecology of visible and invisible work. Computer supported cooperative work (CSCW) 8, 1-2 (1999), 9-30.

[63] A Strauss, S Fagerhaugh, B Suczek, and C Wiener. 1985. Social Organization of Medical Work University of Chicago Press. Chicago, IL (1985).

[64] Lucy Suchman. 1995. Making work visible. Commun. ACM 38, 9 (1995), 56-64.

[65] Charlotte Tang, Yunan Chen, Karen Cheng, Victor Ngo, and John E. Mattison. 2018. Awareness and handoffs in home care: coordination among informal caregivers. Behaviour \& Information Technology 37, 1 (2018), 66-86. https: //doi.org/10.1080/0144929X.2017.1405073

[66] Matthieu Tixier and Myriam Lewkowicz. 2015. Looking for respite and support: Technological opportunities for spousal caregivers. In Proceedings of the 33rd annual ACM conference on human factors in computing systems. ACM, 1155-1158.

[67] Matthieu Tixier and Myriam Lewkowicz. 2016. " Counting on the Group" Reconciling Online and Offline Social Support among Older Informal Caregivers. In Proceedings of the 2016 CHI Conference on Human Factors in Computing Systems. 3545-3558.

[68] Tiffany C Veinot, Hannah Mitchell, and Jessica S Ancker. 2018. Good intentions are not enough: how informatics interventions can worsen inequality. Fournal of the American Medical Informatics Association 25, 8 (2018), 1080-1088.

[69] Sheryl L Walter, Scott E Seibert, Daniel Goering, and Ernest H O’Boyle. 2019. A tale of two sample sources: Do results from online panel data and conventional data converge? Journal of Business and Psychology 34, 4 (2019), 425-452.

[70] Jeremy Warner and Philip J. Guo. 2017. CodePilot: Scaffolding End-to-End Collaborative Software Development for Novice Programmers. In Proceedings of the 2017 CHI Conference on Human Factors in Computing Systems (Denver, Colorado, USA) (CHI '17). Association for Computing Machinery, New York, NY, USA, 1136-1141. https://doi.org/10.1145/3025453.3025876

[71] Ryan Wedoff, Lindsay Ball, Amelia Wang, Yi Xuan Khoo, Lauren Lieberman, and Kyle Rector. 2019. Virtual Showdown: An Accessible Virtual Reality Game with Scaffolds for Youth with Visual Impairments. In Proceedings of the 2019 CHI Conference on Human Factors in Computing Systems (Glasgow, Scotland Uk) (CHI '19). Association for Computing Machinery, New York, NY, USA, 1-15. https://doi.org/10.1145/3290605.3300371

[72] Rainer Winkler, Sebastian Hobert, Antti Salovaara, Matthias Söllner, and Jan Marco Leimeister. 2020. Sara, the Lecturer: Improving Learning in Online Education with a Scaffolding-Based Conversational Agent. In Proceedings of the 2020 CHI Conference on Human Factors in Computing Systems (Honolulu, HI, USA) (CHI '20). Association for Computing Machinery, New York, NY, USA, 1-14. https://doi.org/10.1145/3313831.3376781

[73] Naomi Yamashita, Hideaki Kuzuoka, Keiji Hirata, and Takashi Kudo. 2013. Understanding the conflicting demands of family caregivers caring for depressed family members. In Proceedings of the SIGCHI Conference on Human Factors in Computing Systems. ACM, 2637-2646.

[74] Naomi Yamashita, Hideaki Kuzuoka, Keiji Hirata, Takashi Kudo, Eiji Aramaki, and Kazuki Hattori. 2017. Changing Moods: How Manual Tracking by Family Caregivers Improves Caring and Family Communication. In Proceedings of the 2017 CHI Conference on Human Factors in Computing Systems (Denver, Colorado, USA) (CHI '17). ACM, New York, NY, USA, 158-169. https://doi.org/10.1145/ 3025453.3025843

[75] Naomi Yamashita, Hideaki Kuzuoka, Takashi Kudo, Keiji Hirata, Eiji Aramaki, and Kazuki Hattori. 2018. How Information Sharing about Care Recipients by Family Caregivers Impacts Family Communication. In Proceedings of the 2018 CHI Conference on Human Factors in Computing Systems - CHI '18. ACM Press, New York, New York, USA, 1-13. https://doi.org/10.1145/3173574.3173796 